\title{
L'ingénierie climatique face au réchauffement climatique : solution d'avenir ou fuite en avant?
}

\author{
Olivier Boucher \\ Laboratoire de météorologie dynamique, Institut Pierre-Simon Laplace, \\ CNRS/UPMC, Paris, France
}

\section{Résumé}

L'ingénierie climatique s'est invitée dans le débat sur le changement climatique comme une alternative ou un complément aux efforts de réduction des émissions de gaz à effet de serre et aux politiques d'adaptation aux impacts. Ce concept relativement récent recouvre une large gamme de techniques visant à modifier l'environnement, de manière délibérée et à grande échelle, pour contrecarrer le changement climatique. Cet article se propose de passer en revue ces techniques et d'engager une réflexion sur leurs potentiels, leurs viabilités et leurs risques.

\section{Abstract}

Is climate engineering an opportunity or a threat in the context of global warming?

Climate engineering has entered the debate on climate change as a potential alternative or complement to mitigation and adaptation climate policies. This relatively new concept encompasses a large range of techniques aiming to the deliberate and large-scale modification of the environment in order to alleviate the impacts of climate change. This article will classify and discuss these techniques in terms of their potential, viability and risks. a réalité du changement climatique est maintenant bien établie. Outre le réchauffement des températures de surface observé depuis le début de l'ère industrielle, un faisceau d'indices témoigne du réchauffement en cours : diminution régulière de la glace de mer dans l'océan Arctique, fonte des glaciers continentaux, dégel du permafrost, refroidissement de la stratosphère... Le réchauffement observé ne peut être expliqué à partir des seuls facteurs naturels connus : il faut prendre en compte le forçage radiatif lié à l'augmentation anthropique des gaz à effet de serre pour reproduire l'évolution de la température de surface dans les modèles climatiques (Hegerl et al., 2007). Il n'en reste pas moins que l'évolution future du climat ne peut pas être prévue avec certitude. Les projections existantes reposent sur l'utilisation de modèles climatiques qui représentent de manière imparfaite la complexité du système climatique. C'est pourquoi différents modèles de climat prédisent des degrés différents de réchauffement climatique pour un scénario de gaz à effet de serre donné. Une partie de ces incertitudes provient de notre manque de connaissances sur les rétroactions climatiques et, en particulier, sur la réponse des nuages au changement climatique. Malgré plusieurs décennies de recherches, la sensibilité climatique reste dans une fourchette allant de 1,5 à $4,5^{\circ} \mathrm{C}$ pour un doublement de la concentration atmosphérique en dioxyde de carbone (qui correspond à un forçage radiatif d'environ 3,7 W. $\mathrm{m}^{-2}$ ). Malheureusement, il est difficile de contraindre les modèles et la sensibilité climatique à partir des observations. Une partie du réchauffement climatique dû aux gaz à effet de serre anthropiques a été masquée par l'effet réfléchissant des aérosols, eux aussi issus des activités humaines. On peut aussi bien réconcilier le réchauffement observé avec un effet refroidissant important des aérosols et une sensibilité climatique élevée qu'avec un effet refroidissant des aérosols beaucoup plus faible et une sensibilité climatique moins élevée (Kiehl, 2007). C'est pourquoi les projections climatiques sont le plus souvent présentées de manière probabiliste afin d'inclure les incertitudes sur les scénarios futurs d'émissions, la réponse des cycles biogéochimiques à ces émissions et la réponse du climat au changement de composition chimique de l'atmosphère. Le Groupe intergouvernemental d'experts sur l'évolution du climat (GIEC) a estimé que l'augmentation de température moyenne à la surface du globe à la fin du XXI ${ }^{\mathrm{e}}$ siècle se situera dans une fourchette allant de 1,1 à $6,4{ }^{\circ} \mathrm{C}$ (IPCC, 2007).

Les impacts climatiques pour un réchauffement donné, et la capacité des sociétés à s'y adapter, sont également incertains. Une limite de $2{ }^{\circ} \mathrm{C}$ est souvent donnée comme étant le seuil audelà duquel le changement climatique devient dangereux, mais les bases scientifiques pour une limite de $2^{\circ} \mathrm{C}$ et 1'existence même d'un seuil à partir duquel le changement climatique deviendrait excessivement dangereux sont ténues. Il est néanmoins acquis qu'un réchauffement moyen global de $2{ }^{\circ} \mathrm{C}$ aura des conséquences importantes sur les écosystèmes et les sociétés, 
avec un impact accru pour les pays les plus pauvres qui restent très vulnérables aux aléas climatiques. Les impacts climatiques d'un réchauffement moyen global de $4{ }^{\circ} \mathrm{C}$ ou plus sont substantiels et de nature à modifier profondément la société humaine (New et al., 2011). Il est probable que c'est non seulement l'amplitude du réchauffement climatique mais aussi la vitesse de ce changement qui vont déterminer le coût des politiques d'adaptation et des impacts climatiques résiduels auxquels la société humaine ne pourra pas s'adapter.

Une partie du réchauffement climatique futur est d'ores et déjà inévitable. D'abord, parce que ni le cycle du carbone ni le climat ne sont à l'équilibre. D'une part, les concentrations en dioxyde de carbone $\left(\mathrm{CO}_{2}\right)$ continueraient d'augmenter si les émissions étaient stabilisées à leurs valeurs actuelles. D'autre part, la température du globe continuerait d'augmenter pendant plusieurs décennies si les concentrations en gaz à effet de serre étaient stabilisées. Ensuite, parce que les émissions de gaz à effet de serre dues à la combustion de combustibles fossiles vont sans doute continuer à augmenter dans les années, voire les décennies, qui viennent. Davis et al. (2010) ont fait l'inventaire des infrastructures existantes qui dépendent des combustibles fossiles. Ils ont estimé que leur utilisation jusqu'à leur mise à la retraite programmée produira des émis-

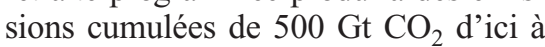
2060, soit un réchauffement de l'ordre de 1,1 à $1,4^{\circ} \mathrm{C}$. Enfin, la diminution des émissions d'aérosols dans le futur plus ou moins proche, en raison des politiques d'amélioration de qualité de l'air, va atténuer l'effet refroidissant dont la planète bénéficiait jusque-là (Boucher, 1997).

Il y a néanmoins des raisons d'espérer malgré ces constats funestes, et il ne faut pas oublier que le futur de la planète reste entre nos mains. Il existe des scénarios qui permettent de limiter les émissions de gaz à effet de serre à un niveau plus acceptable pour la société humaine. Ces scénarios reposent sur une meilleure efficacité énergétique, le développement des énergies renouvelables telles que l'éolien, le solaire ou la biomasse, des technologies de capture et de stockage du $\mathrm{CO}_{2}$ pour les centrales thermiques fonctionnant aux énergies fossiles, ou encore une utilisation accrue de l'énergie nucléaire. Meinshausen et al. (2011) ont montré que les nouveaux scénarios les plus ambitieux reposaient sur un recours important aux biocarburants associé à des techniques de capture et de stockage du $\mathrm{CO}_{2}$. Réduire les émissions de certaines espèces à courte durée de vie, comme le carbone-suie ou les précurseurs de l'ozone troposphérique, permettrait aussi de contenir le réchauffement climatique à plus courte échéance (Shindell et al., 2012). Il est à noter que tous ces scénarios reposent en général sur le choix d'une valeur intermédiaire de la sensibilité climatique. Si le climat est fort sensible aux perturbations radiatives, les efforts à consentir pour stabiliser le climat à un niveau donné seront plus importants.

Les économistes continuent de débattre de l'importance relative à donner aux politiques d'adaptation et d'atténuation du changement climatique. Pas assez d'atténuation et les conséquences du changement climatique, qui seront largement supportées par les générations futures, deviendraient très néfastes. Trop d'atténuation, trop vite, et l'économie pourrait souffrir et induire une diminution du niveau de vie des populations. Le débat se situe à la fois sur le plan économique (comment minimiser les coûts de la réponse à apporter au changement climatique ?), sur le plan de la gestion des incertitudes et des risques (jusqu'à quel point doit-on s'assurer d'éviter un changement climatique aux impacts élevés mais improbables ?), et sur le plan éthique (comment répartir l'effort à produire entre les peuples et les générations ?). Les politiques climatiques de réduction d'émissions n'ont jusqu'à maintenant enregistré qu'un succès très relatif. Le protocole de Kyoto n'a pas été ratifié par tous les pays développés. Il n'a pas permis de stopper, ni même de ralentir, la croissance des émissions mondiales de gaz à effet de serre. Compte tenu des incertitudes sur la sensibilité climatique, on sait qu'il faut que les émissions mondiales atteignent leur maximum d'ici une décennie pour qu'il $\mathrm{y}$ ait une chance raisonnable que le réchauffement moyen reste sous les $2{ }^{\circ} \mathrm{C}$ (Rogelj et al., 2011). Cela nécessite d'inverser la tendance des émissions de $\mathrm{CO}_{2}$ qui continuent d'augmenter (Friedlingstein et al., 2010).

\section{Ingénierie climatique}

C'est dans ce contexte de risques et d'incertitudes que certains ont proposé d'appliquer au problème du changement climatique des solutions que l'on peut qualifier de non conventionnelles et que l'on désigne collectivement sous le nom d'ingénierie climatique (ou parfois ingénierie planétaire). Il n'existe pas de définition consensuelle de l'ingénierie climatique. Le concept n'est pas nouveau et les propositions mises en avant visaient initialement à réchauffer certaines régions inhospitalières telles que l'Arctique. L'idée d'une manipulation du climat, pour compenser le changement climatique dû à l'homme, remonte à 1965 (President's Science Advisory Committee, 1965) et a commencé à être reprise dans les années 1970 (Kellogg et Schneider, 1974). Le terme de géo-ingénierie luimême est apparu dans les années 1970 (Marchetti, 1977), mais dans le contexte d'un stockage du $\mathrm{CO}_{2}$ dans l'océan, et sa signification a évolué depuis. Aujourd'hui, on considère habituellement que relève de l'ingénierie climatique toute technique de manipulation délibérée et à grande échelle de l'environnement, dont le but est de contrecarrer le réchauffement climatique (Keith, 2000 ; IPCC, 2011). Cette définition exclut, par exemple, le refroidissement induit par les émissions actuelles d'aérosols, car celles-ci ne sont pas intentionnelles, ou les tentatives de modification artificielle du temps, dont l'effet recherché est de petite échelle et de courte durée.

Schématiquement, on peut regrouper les techniques d'ingénierie climatique en deux grandes catégories (figure 1). La première englobe les techniques qui visent à diminuer la concentration de $\mathrm{CO}_{2}$ présent dans l'atmosphère, soit en le capturant, soit en manipulant les grands cycles biogéochimiques, pour augmenter le puits atmosphérique de carbone (figure 2). Ces techniques sont qualifiées de techniques d'extraction $d u$ dioxyde de carbone atmosphérique (1). Avant de les discuter dans le détail, il est important de remarquer que la frontière entre ingénierie climatique, atténuation et adaptation n'est pas claire. Selon la définition utilisée par le GIEC, l'atténuation englobe tous les moyens visant à réduire les émissions à la source ou à augmenter les puits des gaz à effet de serre anthropiques (IPCC, 2007). La plupart des techniques d'extraction du $\mathrm{CO}_{2}$ atmosphérique relèvent à la fois de l'atténuation du changement climatique et de l'ingénierie climatique. Il serait donc utile de chercher à mieux classifier les réponses à apporter au changement climatique. La seconde catégorie de techniques consiste à intervenir sur le bilan radiatif autrement qu'en réduisant la concentration de $\mathrm{CO}_{2}$. On peut rendre la planète plus réfléchissante de

(1) Carbon Dioxide Removal, en anglais. 


\section{Ingénierie climatique planétaire

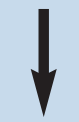

Modification du bilan radiatif<smiles>C[13CH]</smiles>

Extraction des gaz à effet de serre à longue durée de vie de l'atmosphère<smiles>CCCCCC(C)C</smiles>

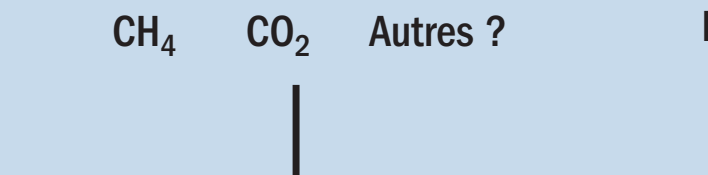

Réduction de l'effet de serre naturel

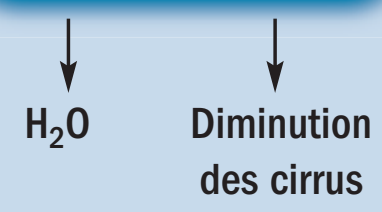

Redistribution de l'énergie dans le système climatique

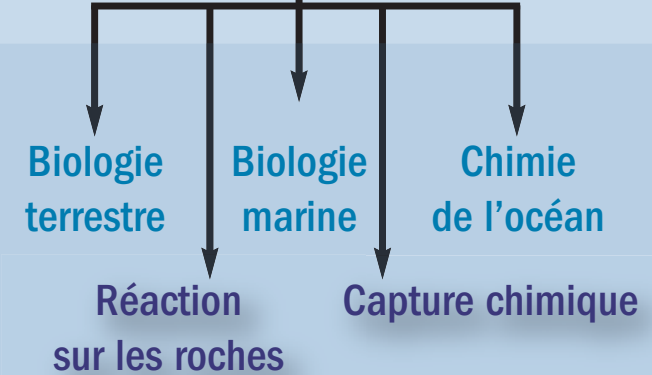

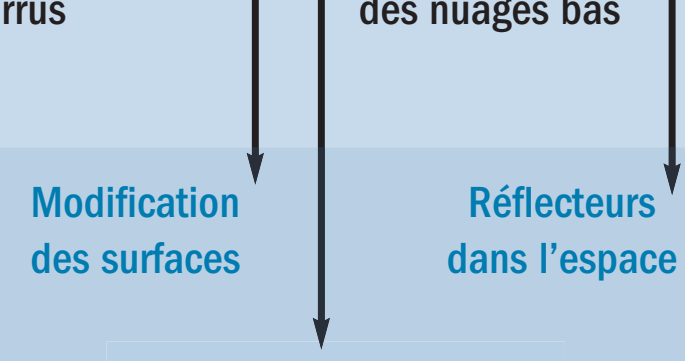

Injection d'aérosols stratosphériques

Figure 1 - Schéma classifiant les différentes méthodes d'ingénierie climatique. On peut regrouper les techniques d'ingénierie climatique en deux grandes catégories : gestion du rayonnement atmosphérique (soit par réduction du rayonnement solaire absorbé, soit par réduction de l'effet de serre naturel) et extraction des gaz à effet de serre à longue durée de vie de l'atmosphère. La troisième catégorie, qui regroupe les techniques visant à redistribuer l'énergie dans le système climatique, n'est guère envisagée et n'est pas discutée dans cet article.

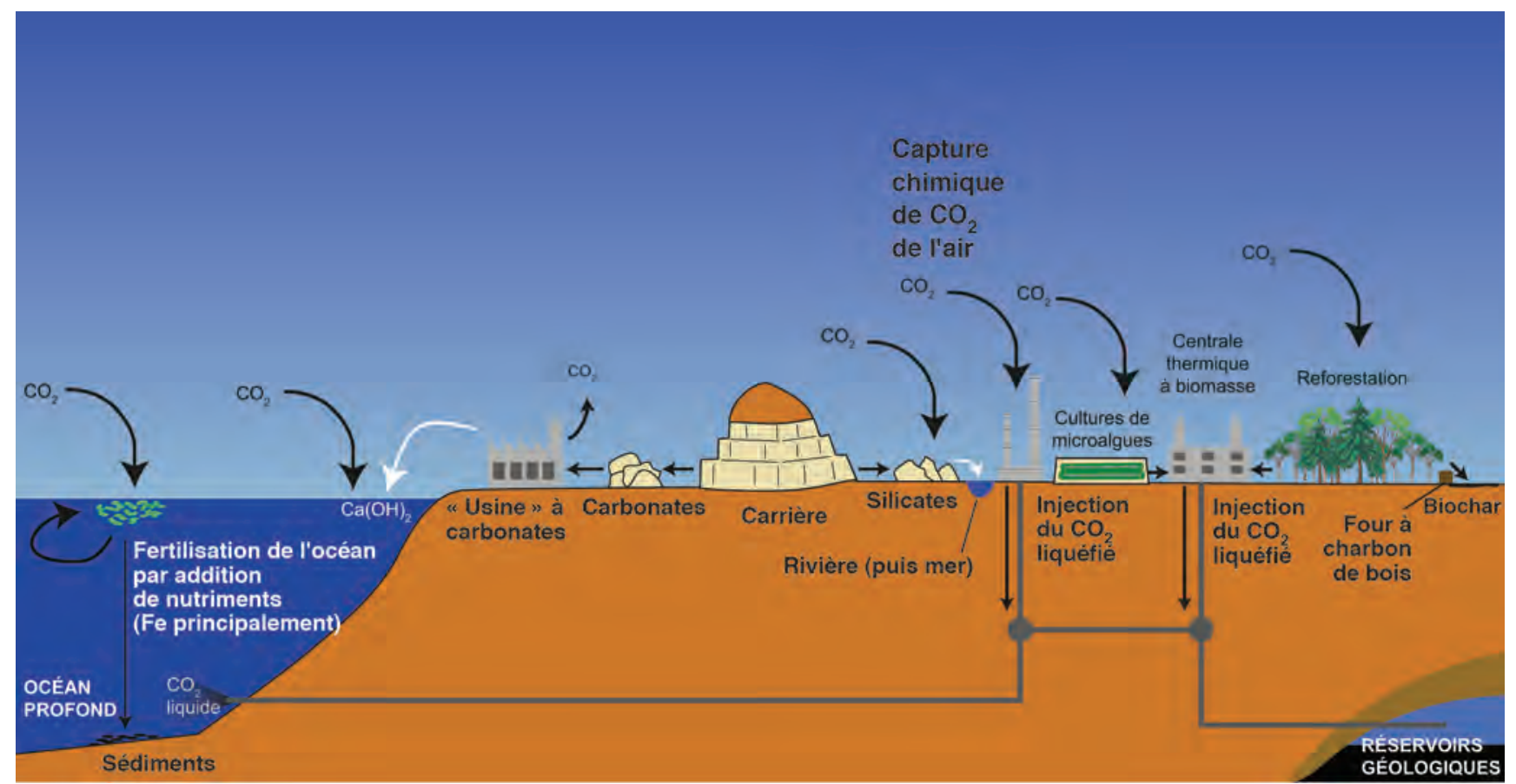




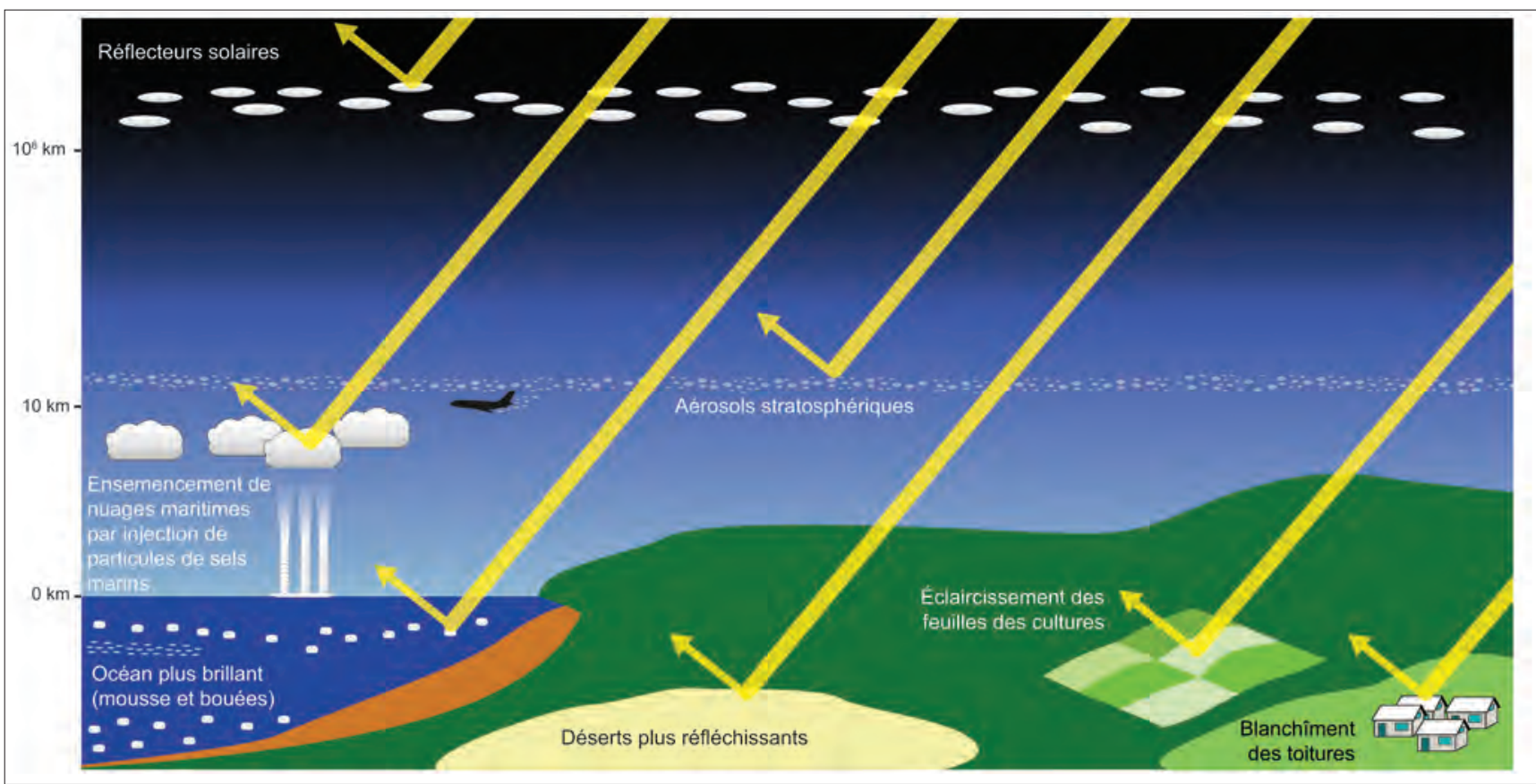

Figure 3 - Représentation schématique des différentes méthodes de gestion du rayonnement solaire. (C Olivier Boucher - Graphisme : Marc Jamous (IPSL-UVSO)).

manière artificielle, par exemple en plaçant des réflecteurs solaire entre le Soleil et la Terre (au point de Lagrange L1), en injectant des aérosols dans la stratosphère, en rendant les nuages plus brillants, ou en augmentant artificiellement la réflectivité des surfaces continentales ou maritimes (figure 3). On peut aussi faire entrer dans cette catégorie d'éventuelles techniques visant à diminuer la couverture ou l'épaisseur des nuages hauts, de type cirrus, dont on sait qu'ils ont un effet réchauffant sur la planète, ou visant à diminuer la concentration en vapeur d'eau, le plus important des gaz à effet de serre dans l'atmosphère, dans la haute troposphère. On désigne ces techniques sous l'appellation de gestion $d u$ rayonnement atmosphérique(1).

\section{Extraction du dioxyde de carbone atmosphérique}

Seule une moitié environ du dioxyde de carbone émis dans l'atmosphère par les activités anthropiques y reste. L'autre moitié est absorbée par les surfaces continentales et les océans. Au-dessus des terres émergées, la végétation utilise le $\mathrm{CO}_{2}$ pour la photosynthèse. On estime que l'effet de fertilisation par le dioxyde de carbone a augmenté la photosynthèse et qu'il en résulte une

(1) Solar radiation management ou Atmospheric radiation management, en anglais. augmentation de la quantité de carbone stocké dans la végétation et les sols (Friedlingstein et al., 2006). Cet effet de fertilisation est sans doute limité dans le temps et pourrait décroître quand les nutriments (azote et phosphore en premier lieu) viendraient à manquer (Thornton et al., 2007). L'autre puits de carbone est dû à la dissolution du dioxyde de carbone dans l'eau de mer et à sa dissociation en ions bicarbonate $\left(\mathrm{CO}_{3}{ }^{2-}\right)$ et carbonate $\left(\mathrm{HCO}_{3}^{-}\right)$. Lorsque la concentration atmosphérique en $\mathrm{CO}_{2}$ augmente, l'équilibre entre l'atmosphère et l'océan est déplacé et une plus grande quantité de $\mathrm{CO}_{2}$ peut se dissoudre. Cet effet sature au fur et à mesure que la concentration en $\mathrm{CO}_{2}$ atmosphérique augmente, car la concentration en $\mathrm{CO}_{2}$ dissous mais non dissocié augmente plus vite que la concentration totale en carbone dissous dans l'eau. Cet effet de saturation est connu sous le nom d'effet Revelle. Le phytoplancton, qui est à la base de la chaîne alimentaire dans l'océan, consomme aussi du carbone pour sa photosynthèse, mais l'écrasante majorité du carbone photosynthétisé est recyclée dans l'océan quand le plancton meurt, et seule une petite fraction de carbone est exportée dans les sédiments. Sur des échelles de temps plus longues, il faut prendre en compte les processus plus lents comme la réaction chimique de l'acide carbonique $\mathrm{H}_{2} \mathrm{CO}_{3}$ sur les roches silicatées pour former du carbonate de calcium $\left(\mathrm{CaCO}_{3}\right)$. Compte tenu de l'importance de ces puits de carbone atmosphérique, on peut légitimement se demander dans quelle mesure on pourrait s'aider ou s'inspirer de la nature pour séquestrer une quantité plus importante du carbone émis par l'utilisation des énergies fossiles.

\section{La solution biologique}

On peut utiliser la végétation de plusieurs manières pour stocker du carbone. La manière la plus simple consiste à le stocker dans la végétation elle-même et, par voie de conséquence, dans les sols au fur et à mesure que l'humus se forme. Une manière d'obtenir ce résultat consiste à développer les surfaces boisées ; cette solution s'appelle reforestation ou afforestation, selon qu'elle utilise des surfaces qui ont déjà été boisées dans le passé ou non. Dans ce cas, le puits de carbone n'est que transitoire (typiquement de quelques décennies à un siècle) puisqu'une fois arrivée à maturité, la forêt émettra autant de carbone qu'elle en absorbera. Le carbone stocké reste toutefois vulnérable aux effets du changement climatique ou à la suppression éventuelle de la forêt.

On peut rechercher des solutions qui permettraient de stocker le carbone de manière plus durable. Certains proposent de brûler la biomasse pour en tirer une sorte de charbon de bois à stocker dans le sol, un composant connu sous le nom de biochar. Cette technique permet non seulement de récupérer une fraction de l'énergie contenue dans la biomasse et de stocker du carbone, mais 
elle pourrait aussi servir à augmenter la fertilité de certains sols (Lehmann et al., 2006). Une utilisation plus complète de l'énergie contenue dans la biomasse ne peut extraire du $\mathrm{CO}_{2}$ de l'atmosphère que si la culture de la biomasse (bois ou cultures énergétiques(1) ${ }^{(1)}$ est couplée à une technique de capture et de séquestration du carbone(2) en aval de la combustion. On peut signaler au passage la difficulté à classifier les techniques d'ingénierie climatique : ni les techniques de séquestration du $\mathrm{CO}_{2}$ provenant des combustibles fossiles, ni l'utilisation de la biomasse renouvelable ne sont, en général, considérées comme relevant de l'ingénierie climatique, mais la combinaison des deux l'est car elle permet de réduire la concentration de $\mathrm{CO}_{2}$ dans l'atmosphère.

Diverses études ont essayé de quantifier le potentiel de séquestration de ces différentes options (Lenton et Vaughan, 2009 ; Royal Society, 2009). Ces potentiels ne sont pas négligeables (voir encadré cidessous), et le rôle des écosystèmes terrestres dans le stockage du carbone mérite d'être étudié plus avant. Il est important de noter néanmoins que les potentiels de ces différentes techniques ne s'additionnent pas nécessairement les uns aux autres, car celles-ci entrent en compétition pour l'utilisation des sols. L'utilisation de la biomasse à grande échelle pour contrer le changement climatique pose donc le problème des poids respectifs à donner à l'agriculture alimentaire, aux agro-carburants, aux forêts et à la protection de la biodiversité.

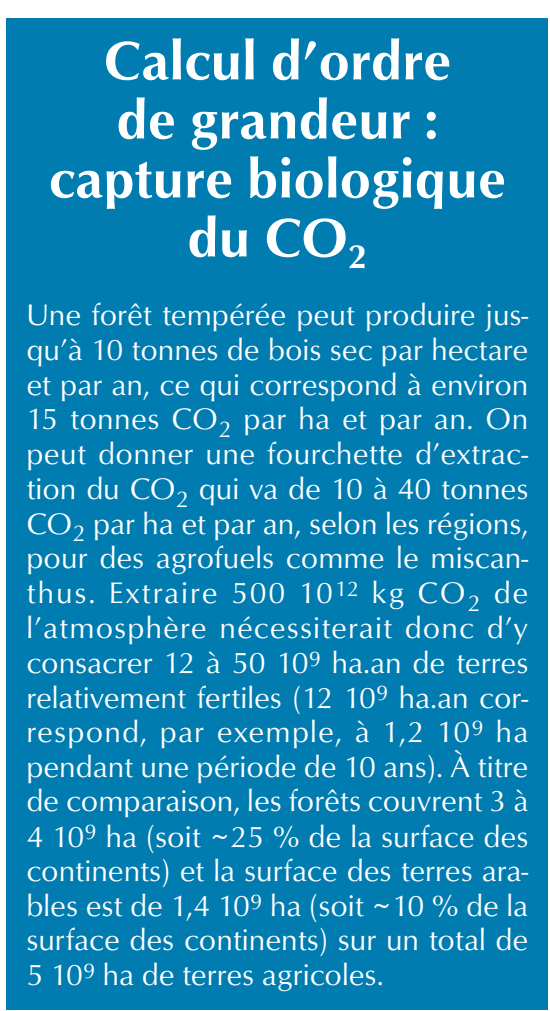

Réconcilier tous ces objectifs par une agriculture plus intensive pourrait nécessiter l'utilisation de fertilisants et de pesticides dont il faut également prendre en compte les effets secondaires sur l'environnement.

\section{La solution géologique}

Il est aussi envisageable d'essayer d'accélérer les processus physico-chimiques naturels qui transfèrent du $\mathrm{CO}_{2}$ vers des réservoirs de carbone plus stables (carbone dissous dans l'océan, carbone organique dans les sédiments marins ou encore carbonate de calcium dans les roches et les sédiments). À l'exception du carbone dissous dans l'océan de surface, les mécanismes d'échange de carbone vers les réservoirs géologiques sont notoirement lents et ne représentent qu'une petite fraction du flux de carbone sortant de l'atmosphère. La question est donc de savoir si l'on peut accélérer ces processus. Là encore, plusieurs techniques ont été proposées.

La molécule de $\mathrm{CO}_{2}$ est le produit final des chaînes d'oxydation dans l'atmosphère. Bien qu'elle soit très stable dans l'atmosphère, elle n'en reste pas moins une molécule réactive car le dioxyde de carbone est un acide faible en solution. La dissolution des roches silicatées consomme deux molécules de $\mathrm{CO}_{2}$ par molécule de $\mathrm{CaSiO}_{3}$, mais une molécule de $\mathrm{CO}_{2}$ est ensuite relâchée lors de la précipitation d'un carbonate quand le milieu est sursaturé par rapport au $\mathrm{CaCO}_{3}$. Cette réaction, due à Urey (1952), peut s'écrire schématiquement :

$$
\mathrm{CaSiO}_{3}+\mathrm{CO}_{2} \rightarrow \mathrm{CaCO}_{3}+\mathrm{SiO}_{2}
$$

On peut donc imaginer favoriser cette réaction en extrayant des roches silicatées. Une variante de cette méthode consisterait à jeter les matériaux dissous dans l'océan avec l'avantage de stocker deux molécules de $\mathrm{CO}_{2}$ par molécule de silicate :

$$
\begin{gathered}
\mathrm{CaSiO}_{3}+2 \mathrm{CO}_{2}+\mathrm{H}_{2} \mathrm{O} \rightarrow \\
\mathrm{Ca}^{2+}+2 \mathrm{HCO}_{3}^{-}+\mathrm{SiO}_{2}
\end{gathered}
$$

Cela n'est pas impossible mais nécessiterait d'extraire et de broyer de la roche à très grande échelle pour compenser la lenteur de la réaction chimique, même si certaines roches, comme les olivines ou les verres basaltiques, présentent l'avantage d'une vitesse de dissolution relativement élevée (Schuiling et de Boer, 2011).

L'océan étant un réservoir de carbone bien plus important que l'atmosphère (avec respectivement 38000 et $80010^{12} \mathrm{~kg} \mathrm{C}$ ), on peut se demander si sa capacité peut être dopée. Là encore, la réponse est oui, mais à condition de pouvoir modifier la composition chimique de l'eau de mer à grande échelle. Kheshgi (1995) a suggéré d'introduire du carbonate de sodium $\left(\mathrm{Na}_{2} \mathrm{CO}_{3}\right)$ ou de l'hydroxyde de calcium $\left(\mathrm{Ca}(\mathrm{OH})_{2}\right.$ ou chaux éteinte) dans l'océan de manière à en augmenter le $\mathrm{pH}$ et à déplacer l'équilibre chimique entre $\mathrm{CO}_{2}, \mathrm{HCO}_{3}^{-}$ et $\mathrm{CO}_{3}{ }^{2-}$ vers plus de carbonates. Cette technique permettrait en même temps de remédier à l'acidification des océans. Cependant, les réserves naturelles de carbonate de sodium sont réduites et l'hydroxyde de calcium n'existe pas à l'état naturel sur Terre. Il faudrait donc produire ce dernier à très grande échelle, à partir de carbonate de calcium, par décomposition thermique :

$$
\mathrm{CaCO}_{3}+178 \mathrm{~kJ} / \mathrm{mol} \rightarrow \mathrm{CaO}+\mathrm{CO}_{2}
$$

suivie de

$$
\mathrm{CaO}+\mathrm{H}_{2} \mathrm{O} \rightarrow \mathrm{Ca}(\mathrm{OH})_{2}+\text { chaleur }
$$

La première réaction requiert beaucoup d'énergie et produit du $\mathrm{CO}_{2} \ldots$ mais en quantité moindre que le $\mathrm{CO}_{2}$ qui serait ensuite absorbé dans l'océan. Au final, le bilan chimique s'écrit :

$$
\begin{gathered}
\mathrm{CaCO}_{3}+\mathrm{CO}_{2}+\mathrm{H}_{2} \mathrm{O}+\text { énergie } \rightarrow \\
\mathrm{Ca}^{2+}+2 \mathrm{HCO}_{3}^{-}
\end{gathered}
$$

Le site www.cquestrate.com estime qu'une molécule de $\mathrm{CaO}$ (et donc une molécule de $\mathrm{CO}_{2}$ émise dans l'atmosphère) permettrait d'extraire 1,79 molécules de $\mathrm{CO}_{2}$ de 1'atmosphère, soit un gain net de 0,79 molécule de $\mathrm{CO}_{2}$. Les conséquences d'une telle modification de la composition chimique de l'océan sur les écosystèmes marins ne sont pas connues. La durabilité du stockage de carbone dans l'océan n'a pas été évaluée. De plus, la quantité d'énergie nécessaire serait énorme et la modification de la composition chimique de l'océan sans doute irréversible. Il est donc difficile d'être très enthousiaste quant à cette méthode.

\section{La solution technologique}

Compte tenu des effets secondaires possibles des solutions biologique et géologique évoquées ci-dessus, se pose la question d'une éventuelle solution technologique qui permettrait de retirer le $\mathrm{CO}_{2}$ de l'atmosphère sans manipuler l'océan ou les écosystèmes. Les cycles

(1) Energy crop, en anglais.

(2) Carbon Capture and Storage ou CCS, en anglais. 
chimiques décrits ci-dessus peuvent être utilisés en circuit fermé. La molécule de $\mathrm{CO}_{2}$ est soluble et réagit, en particulier, avec de la soude $(\mathrm{NaOH})$ en solution pour former du carbonate de soude. Cette réaction chimique peut être utilisée pour capturer le $\mathrm{CO}_{2}$ atmosphérique qui se dissout dans la solution au fur et à mesure que le $\mathrm{CO}_{2} \mathrm{y}$ est consommé. La solution peut ensuite être mélangée à de la chaux éteinte $\left(\mathrm{Ca}(\mathrm{OH})_{2}\right)$ pour recycler la soude. Le carbonate de calcium qui précipite doit alors être chauffé pour recycler la chaux, et du $\mathrm{CO}_{2}$ pur est obtenu qui doit ensuite être stocké. Le procédé est ancien (Baciocchi et al., 2006 ; Zeman, 2007) et des prototypes ont été construits. Plus que le coût financier, c'est le coût énergétique qu'il faut examiner pour évaluer le potentiel et la pérennité d'une telle méthode.

La thermodynamique nous dit que l'énergie minimale nécessaire pour séparer deux gaz est l'opposé du changement de l'énergie libre de Gibbs quand les deux gaz sont mélangés. Pour un gaz trace comme le dioxyde de carbone, on peut approximer cette énergie par : $\Delta \mathrm{Q}=\mathrm{R} \mathrm{T}(1-\ln \mathrm{x}) / \mathrm{M}$

où $\mathrm{R}$ est la constante universelle des gaz parfaits, $T$ est la température, $M$ est la masse moléculaire du gaz et x est le rapport de mélange en volume du gaz, sous l'hypothèse que $x$ est petit par rapport à 1 (Boucher et Folberth, 2010). L'énergie thermodynamique minimale nécessaire pour séparer 1 mole de $\mathrm{CO}_{2}$ de l'air ambiant, pour lequel le rapport de mélange est de $380 \mathrm{ppm}$ (soit $\mathrm{x}=38010^{-6}$ ), est donc de $22 \mathrm{~kJ}$. On peut estimer que n'importe quel processus réel pour séparer le $\mathrm{CO}_{2}$ consommera au minimum trois fois plus d'énergie que cette énergie minimale (McKay, 2009). On pourrait utiliser le fait que les différents gaz présents dans 1 'atmosphère ont des températures d'ébullition différentes et procéder à une séparation cryogénique, mais le coût énergétique est prohibitif. En fait, la solution chimique évoquée ci-dessus est sans doute la moins énergivore. Zeman (2007) estime le coût énergétique de la séparation chimique à environ $400 \mathrm{~kJ} . m o l^{-1}$, auquel il faut ajouter le coût de la compression et du stockage du $\mathrm{CO}_{2}$. Même si une partie de la chaleur dégagée $\left(180 \mathrm{~kJ}^{\circ} \mathrm{mol}^{-1}\right)$ peut éventuellement être récupérée, le coût total reste environ 20 fois plus élevé que le coût thermodynamique minimum. On peut estimer que supprimer $1 \mathrm{~W} \cdot \mathrm{m}^{-2}$ de forçage radiatif par le $\mathrm{CO}_{2}$ requiert de baisser sa concentration de 380 à $315 \mathrm{ppm}$, soit $50010^{12} \mathrm{~kg} \mathrm{CO} \mathrm{CO}_{2}$ ou encore $1,210^{16} \mathrm{~mol} \mathrm{CO}_{2}$, ce qui nécessiterait d'y consacrer une énergie de $610^{21} \mathrm{~J}$ (voir encadré ci-dessous). Une telle quantité d'énergie représente l'équivalent de 13 ans de notre consommation actuelle d'énergies fossiles (prise égale ici à $1,510^{13} \mathrm{~W}$ ). Et c'est sans compter le $\mathrm{CO}_{2}$ qui ne manquerait pas de dégazer de l'océan si la concentration atmosphérique venait à diminuer. De plus, pour que la capture du $\mathrm{CO}_{2}$ ait un sens, il faut que cette énergie provienne de sources d'énergie ne produisant pas ou peu de $\mathrm{CO}_{2}$. Cela montre que la capture du $\mathrm{CO}_{2}$ n'est véritablement envisageable que si l'humanité dispose d'une source abondante d'énergie décarbonée et bon marché, ce qui ne sera peut-être jamais le cas.

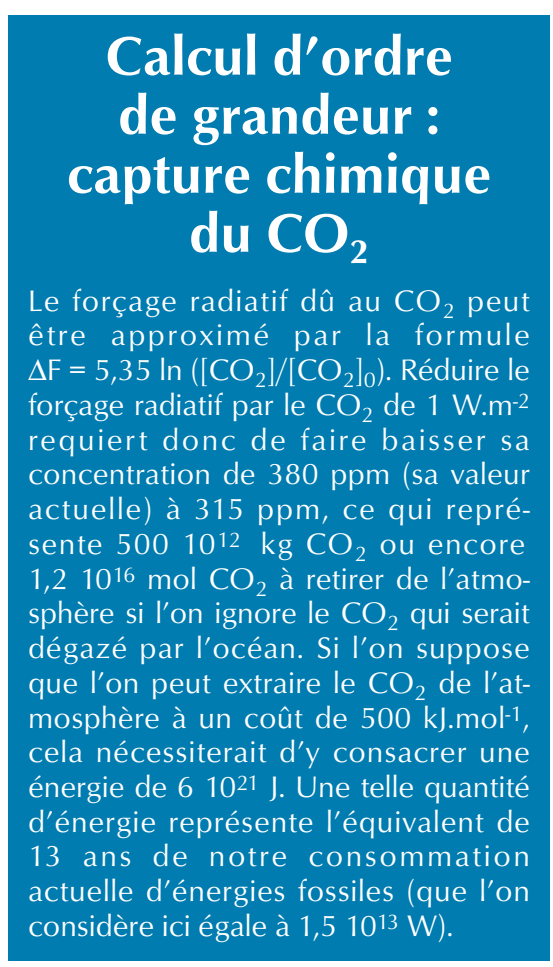

Il existe une autre piste qui n'a pas encore été explorée. Elle consisterait à utiliser des bactéries extrémophiles chimio-autotrophes capables de réduire du $\mathrm{CO}_{2}$ en carbone en présence de fer (Pugh et Umbreit, 2009).

\section{Capturer le $\mathrm{CO}_{2}$ pour en faire quoi ?}

Les méthodes décrites ci-dessus ne stockent pas nécessairement le $\mathrm{CO}_{2}$ de manière définitive. Si le carbone stocké dans les sédiments est probablement stable sur des échelles de temps de l'ordre du millénaire, celui stocké dans la biosphère continentale peut, en revanche, être relâché dans l'atmosphère si la végétation et les sols répondent au changement climatique (Friedlingstein et al., 2006) ou si l'utilisation des sols change. Par exemple, la pérennité du biochar comme moyen de stockage du carbone n'est pas bien connue.

Le $\mathrm{CO}_{2}$ extrait de l'atmosphère, soit par capture à la source lors de la combustion de biocarburants, soit par capture chimique directe dans l'atmosphère, doit être stocké quelque part. Cela peut être sous forme liquide ou solide dans l'océan profond ou dans des couches géologiques profondes comme des aquifères, tel qu'on envisage déjà de le faire pour le $\mathrm{CO}_{2}$ qui pourrait être capturé dans les centrales thermiques. La durée de vie du $\mathrm{CO}_{2}$ ainsi piégé dans les océans ou dans les aquifères dépend de la manière dont il a été injecté et de l'étanchéité des aquifères (IPCC, 2005). D'autres formes de stockage peuvent être envisagées comme, par exemple, du carbone amorphe sous forme de graphite. Dans tous les cas, il faut prendre en compte le fait que la liquéfaction du $\mathrm{CO}_{2}$, ou sa transformation en un composé chimique stable, a un coût énergétique.

À défaut de stocker le $\mathrm{CO}_{2}$, on peut essayer d'en faire un usage industriel. Cela ne réduira pas directement les concentrations atmosphériques (le $\mathrm{CO}_{2}$ capturé se retrouvera dans l'atmosphère) mais permettrait de réduire quelque peu les émissions. Cependant, les applications industrielles du $\mathrm{CO}_{2}$ sont extrêmement limitées au regard des quantités de $\mathrm{CO}_{2}$ présentes dans 1'atmosphère. La capture du $\mathrm{CO}_{2}$ atmosphérique aurait des débouchés plus importants si celui-ci pouvait être reconverti en un produit utile pour l'industrie chimique. Aussi surprenant que cela paraisse, il s'agit là d'un champ de recherche actif qui s'inscrit dans un effort plus vaste visant à comprendre et à reproduire en laboratoire les processus chimiques de la photosynthèse et/ou à réduire le $\mathrm{CO}_{2}$ de manière douce. On peut mentionner ici les résultats de Angamuthu et al. (2010) qui ont réussi à recombiner deux molécules de $\mathrm{CO}_{2}$ en un anion oxalate $\left(\mathrm{C}_{2} \mathrm{O}_{4}{ }^{2-}\right)$ à l'aide d'un catalyseur à base de cuivre. L'anion oxalate peut être « détaché » du catalyseur avec un potentiel électrique relativement faible en comparaison avec le potentiel électrique qui serait nécessaire pour réduire le $\mathrm{CO}_{2}$ directement. Le catalyseur peut alors être recyclé. L'intérêt de ce procédé est qu'il génère une molécule avec deux atomes de carbone, ce qui constitue le premier pas vers des molécules organiques plus 
complexes qui peuvent servir de matière première pour l'industrie chimique. Mais la route reste longue : le catalyseur doit pouvoir être recyclé de nombreuses fois, le produit doit pouvoir être exploité (c'est le cas, par exemple, du méthanol, du formaldéhyde ou de l'acide oxalique), le processus doit être énergétiquement abordable et financièrement rentable. Il faut garder à l'esprit que l'énorme majorité du pétrole que nous consommons est brûlée et que seule une petite fraction est utilisée par l'industrie pétrochimique. La conversion du $\mathrm{CO}_{2}$ à grande échelle n'a donc de sens que si on peut en faire, au final, un combustible à partir d'une énergie renouvelable.

\section{Et le méthane ?}

La recherche sur la capture de gaz à effet de serre s'est principalement focalisée sur le $\mathrm{CO}_{2}$ en raison de sa prédominance sur le forçage radiatif anthropique. Le méthane est le deuxième gaz à effet de serre avec un forçage radiatif de $0,48 \mathrm{~W} \cdot \mathrm{m}^{-2}$, son efficacité radiative est 20 fois plus grande que celle du $\mathrm{CO}_{2}\left(3,710^{-4}\right.$ contre $\left.1,54810^{-5} \mathrm{~W} \cdot \mathrm{m}^{-2} \mathrm{ppbv}^{-1}\right)$. Mais sa concentration atmosphérique est beaucoup plus faible (1,8 ppm au lieu de $380 \mathrm{ppm}$ ), ce qui rend sa capture beaucoup plus difficile. Il n'est pas exclu cependant que l'on puisse augmenter l'intensité des puits de méthane, soit aux travers des pratiques agricoles en oxygénant mieux les sols, soit en convertissant le méthane atmosphérique par catalyse (Boucher et Folberth, 2010 ; de Richter et Caillol, 2011).

\section{Gestion du rayonnement atmosphérique}

La gestion du rayonnement atmosphérique est l'autre grande catégorie de techniques d'ingénierie climatique. La planète ne s'écarte que relativement peu de l'équilibre radiatif entre, d'une part, le rayonnement solaire absorbé par l'atmosphère et la surface terrestre, et, d'autre part, le rayonnement infrarouge qui sort de l'atmosphère vers l'espace. Le climat répond à un déséquilibre radiatif au sommet de l'atmosphère par un réchauffement ou un refroidissement, qui permet d'ajuster le rayonnement infrarouge sortant via une série de rétroactions climatiques. Ces processus sont à la base des variations climatiques naturelles passées et du réchauffement climatique récent. Il est donc indéniable qu'une augmentation artificielle de la réflectivité (ou albédo) de la planète entraînerait un refroidissement qui pourrait compenser le réchauffement (présent et futur) dû aux gaz à effet de serre. Pour chaque W. $\mathrm{m}^{-2}$ de forçage radiatif par les gaz à effet de serre, il suffirait en effet d'augmenter la réflectivité de la planète d'un peu plus de $0,3 \%$ (par exemple, en la faisant passer de $30 \%$ à $30,3 \%$ ) pour maintenir la température moyenne de la planète à peu près constante.

En principe, on pourrait augmenter l'albédo de la planète en injectant de petites particules dans la stratosphère (Crutzen, 2006 ; Keith 2010), en ensemençant les nuages bas au-dessus des océans (Latham, 1990 ; Salter et al., 2008) avec des particules de sels marins, ou encore en augmentant la réflectivité des surfaces continentales (Hamwey, 2007 ; Akbari et al., 2009 ; Ridgwell et al., 2009 ; Irvine et al., 2011) ou des océans (Evans et al., 2010 ; Seitz, 2011), comme illustré sur la figure 3. Le degré de maturité de ces projets d'ingénierie climatique est très variable. Mitchell et Finnegan (2009) ont, quant à eux, proposé d'ensemencer les nuages hauts pour réduire leur couverture et réduire leur effet de serre.

Les éruptions volcaniques, telle que celle du Pinatubo en juin 1991, fournissent la preuve qu'il est possible de refroidir le climat en injectant dans la stratosphère une fraction des gaz soufrés émis quotidiennement dans l'atmosphère par la combustion des combustibles fossiles (charbon, pétrole et gaz). L'efficacité des aérosols stratosphériques à refroidir le climat est relativement élevée car le temps de résidence de ceux-ci est beaucoup plus long dans la stratosphère que dans la troposphère. Il serait possible de refroidir le climat d'environ $1{ }^{\circ} \mathrm{C}$ en injectant, de manière continue, 1,5 mégatonnes de soufre $(\mathrm{S})$ par an dans la stratosphère (à titre de comparaison, les activités industrielles émettent environ 70 mégatonnes de $\mathrm{S}$ par an dans l'atmosphère). On peut estimer que la perte d'énergie solaire induite par les aérosols stratosphériques $\left(\sim 10^{13} \mathrm{~J} / \mathrm{kg}\right)$ est au moins 1 million de fois plus importante que l'énergie nécessaire pour injecter le $\mathrm{S}$ dans la stratosphère $\left(<10^{7} \mathrm{~J} / \mathrm{kg}\right.$, voir encadré ci-dessous). Cependant, on ne peut pas augmenter la charge en aérosols de la stratosphère indéfiniment. Si les aérosols sont trop nombreux, ils coagulent, grossissent et tombent plus rapidement (Rasch et al., 2008), mais on pense qu'il est possible d'injecter assez de soufre pour atteindre un refroidissement de plusieurs degrés et compenser un doublement de la concentration en $\mathrm{CO}_{2}$ (Heckendorn et al., 2009). Pour cela, les quantités de gaz soufrés à injecter dans la stratosphère seraient substantielles (peut-être une dizaine de mégatonnes par an) mais du domaine du possible pour une flotte d'avions de transport. Des ingénieurs travaillent sur des solutions plus pratiques comme celle d'un tube de faible diamètre, de $20 \mathrm{~km}$ de haut, suspendu à un ballon stratosphérique amarré au sol et dans lequel seraient pompés des gaz soufrés : un

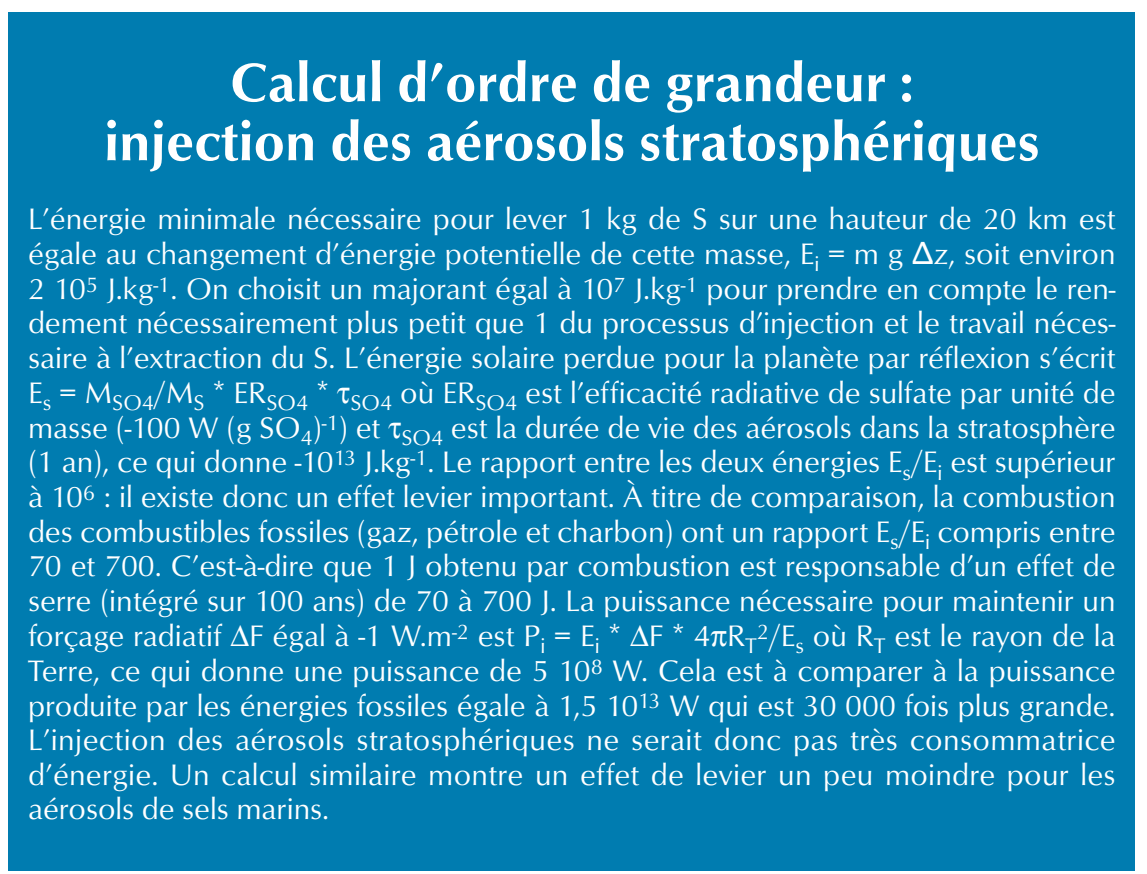


procédé déjà breveté (Davidson et al., 2010) ! Parmi les inconvénients de la méthode des aérosols stratosphériques figure une possible diminution de la couche d'ozone stratosphérique (Tilmes et al., 2008, 2009 ; Robock et al., 2009) et du rayonnement solaire incident à la surface terrestre, lequel est nécessaire à la photosynthèse. Les impacts sur le rayonnement ultraviolet à la surface ne sont pas clairs. Si la diminution de l'ozone stratosphérique augmentait quelque peu le rayonnement ultraviolet à la surface, les aérosols stratosphériques, eux, le réduiraient (Boucher, 2008), si bien que l'effet net n'est pas connu. Les impacts des aérosols stratosphériques sur la photosynthèse et sur la productivité primaire sont également incertains, car les aérosols stratosphériques diminuent le rayonnement solaire à la surface mais ils augmentent la fraction diffuse, ce qui favorise la croissance des plantes (Mercado et al., 2009).

L'ensemencement des nuages maritimes de la couche limite par des sels marins pour les rendre plus brillants a été proposé comme une alternative aux aérosols stratosphériques. Les sels marins pourraient être injectés par des bateaux spécialisés qui pomperaient de l'eau de mer pour la propulser dans l'atmosphère sous forme de petites particules. Un ingénieur anglais est allé jusqu'à dessiner les plans de bateaux futuristes exploitant l'énergie du vent pour diffuser des sels marins dans la couche limite (Salter et al., 2008 ; figure 4). Les effets secondaires sur l'atmosphère elle-même seraient probablement moindres que pour les aérosols

Figure 4 - Vue d'artiste d'un bateau fonctionnant à l'énergie éolienne qui sillonnerait les océans pour injecter des aérosols de sels marins dans la couche limite marine. (D'après Salter et al. (2008). C) John MacNeill).

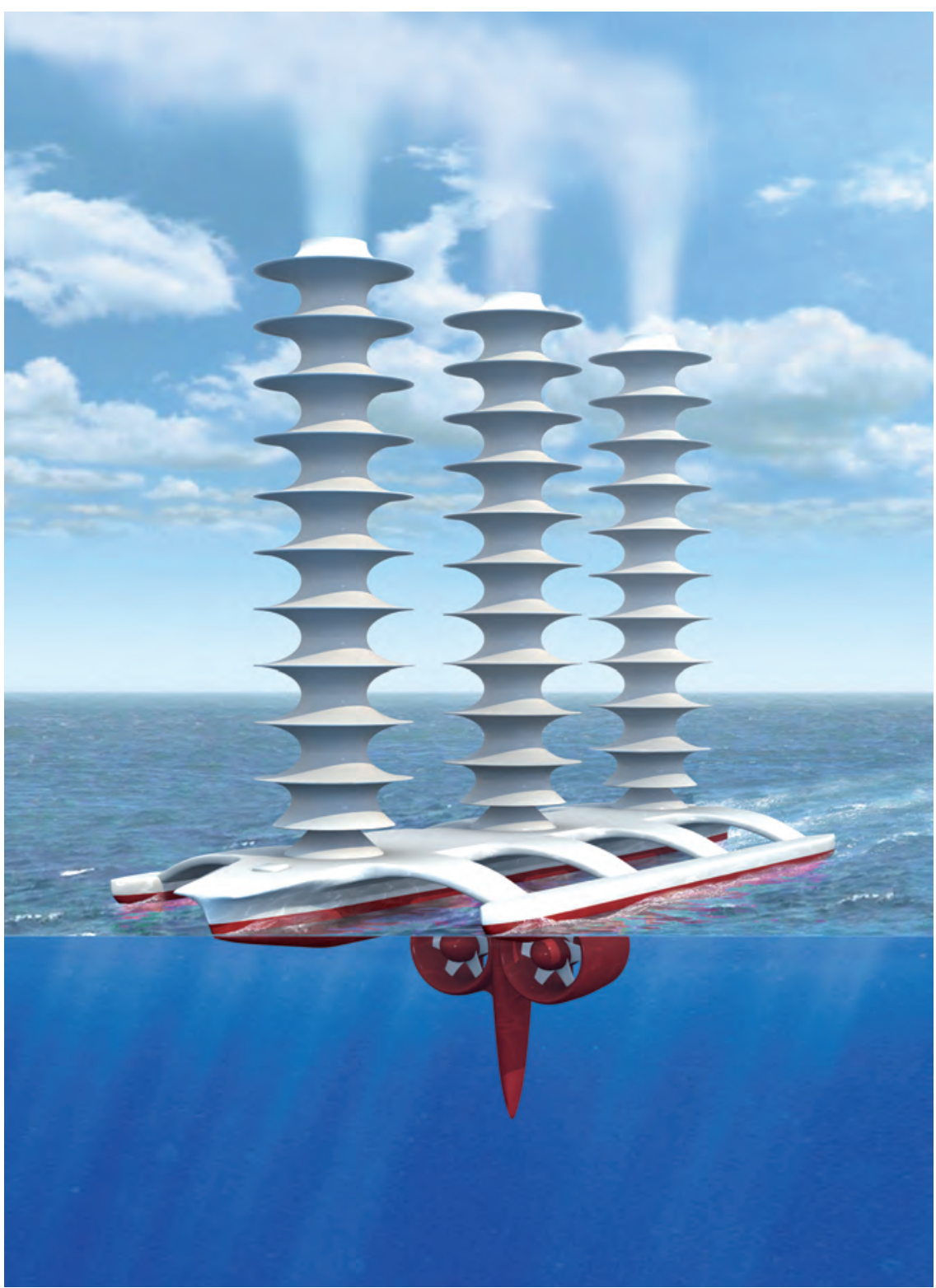

\section{Réponse climatique aux modifications du rayonnement solaire}

Jusqu'à quel point peut-on contrôler le climat? L'objectif de l'ingénierie climatique est de manipuler le climat pour qu'il ressemble au climat préindustriel, au climat actuel ou à un climat jugé optimal pour l'humanité et les écosystèmes dont elle dépend. Mais il est illusoire de vouloir compenser de manière exacte le changement climatique induit 
par l'effet de serre par une augmentation de l'albédo de la planète. Même si la planète gardait la même température moyenne, par le biais d'une compensation entre augmentation de l'effet de serre et augmentation artificielle de la réflectivité de la planète, certaines régions se réchaufferaient et d'autres se refroidiraient. Ce résultat a maintenant été confirmé par de nombreuses études de modélisation (Robock et al., 2008 ; Bala et al., 2008 ; Caldeira et Wood, 2008 ; Jones et al., 2010 ; Irvine et al., 2010 ; Schmidt et al., 2012). La figure 5 a montre, par exemple, la distribution du changement de température simulée par des modèles de climat, dans une expérience idéalisée où le forçage radiatif dû à un quadruplement de la concentration en $\mathrm{CO}_{2}$ a été compensé par une diminution ad hoc de la constante solaire. On peut observer un refroidissement des tropiques et un réchauffement des hautes latitudes, même si ces changements de température sont relativement faibles comparés à ceux dus au quadruplement du $\mathrm{CO}_{2}$ seul.

Il n'en est pas forcément de même du cycle hydrologique et des précipitations. La compensation de l'effet de serre du $\mathrm{CO}_{2}$ par une réduction équivalente de la quantité de rayonnement solaire absorbé modifie profondément le bilan énergétique de l'atmosphère qui s'adapte en ralentissant le cycle hydrologique à l'échelle globale. Les simulations climatiques qui ont été réalisées montrent qu'il y aurait des augmentations et des diminutions régionales de précipitations qui seraient du même ordre de grandeur qu'en présence du réchauffement climatique (figure $5 \mathrm{~b}$; Schmidt et al., 2012). Même les études cherchant à optimiser la distribution de l'ingénierie planétaire simulent un changement climatique résiduel important (Ban-Weiss et Caldeira, 2010 ; Ricke et al., 2010). Il n'est donc pas possible d'affirmer que l'ingénierie climatique assurerait à tous les pays un climat meilleur ou au moins aussi favorable que le climat présent.

De plus, le refroidissement induit par un forçage radiatif essentiellement localisé au-dessus des océans ne se propagerait pas forcément sur tous les continents, $a$ fortiori si ce forçage radiatif est hétérogène spatialement (Jones et al., 2009 ; Alterskjær et al., 2012). C'est pourquoi les techniques visant à augmenter la réflectivité des nuages océaniques ou de l'océan lui-même sont a priori moins intéressantes que l'injection d'aérosols dans la stratosphère.

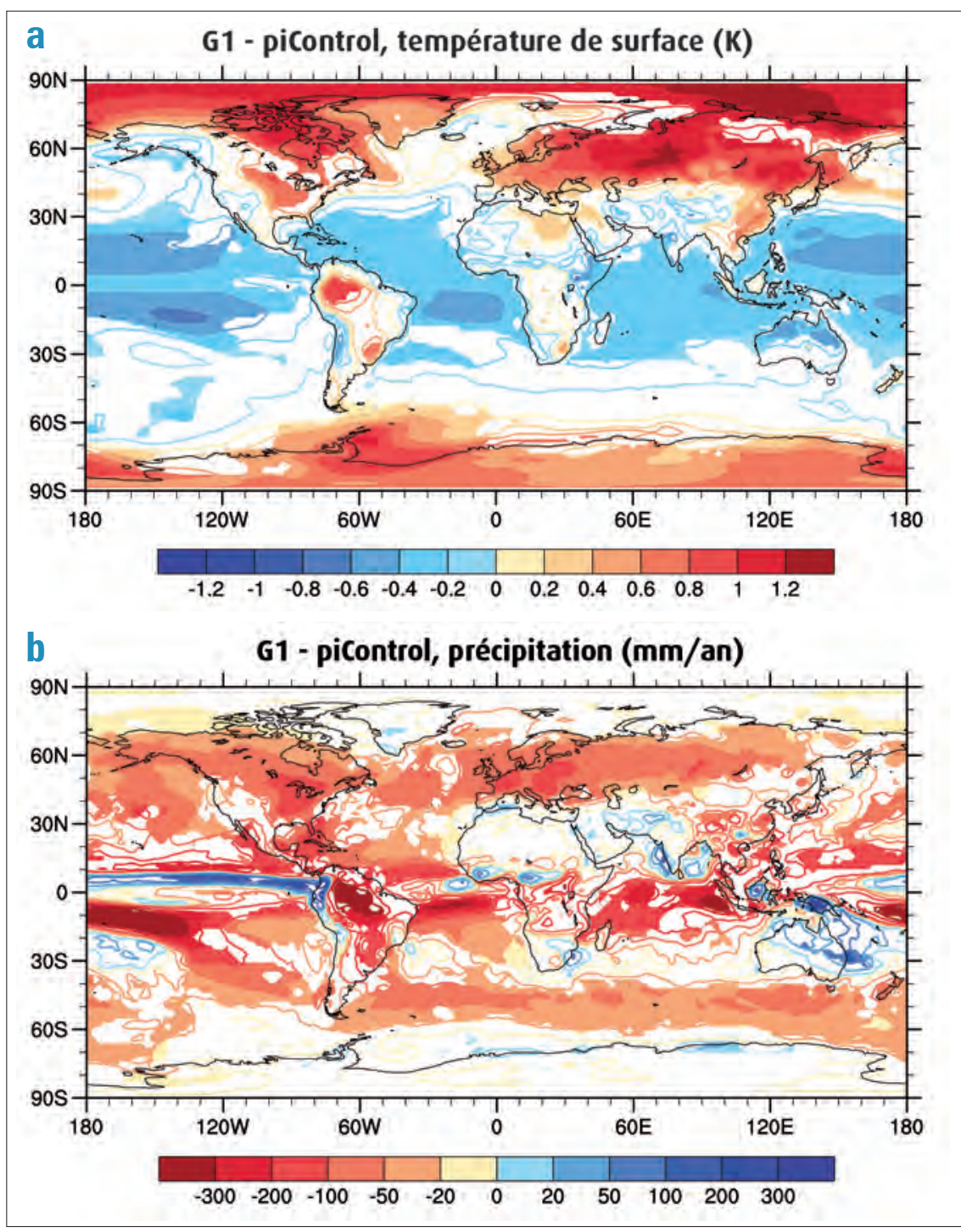

Figure 5 - (a) changement de température $\left({ }^{\circ} \mathrm{C}\right.$ ) et (b) changement de précipitation (mm/an) dans une simulation idéalisée où le forçage radiatif, dû à un quadruplement de la concentration en $\mathrm{CO}_{2}$, a été compensé par une diminution de la constante solaire. On a représenté en couleur les régions où les 4 modèles climatiques ayant réalisé l'expérience simulent un changement de même signe. (D'après Schmidt et al. (2012), suivant le protocole expérimental détaillé dans Kravitz et al. (2011). (C) Creative Common License).

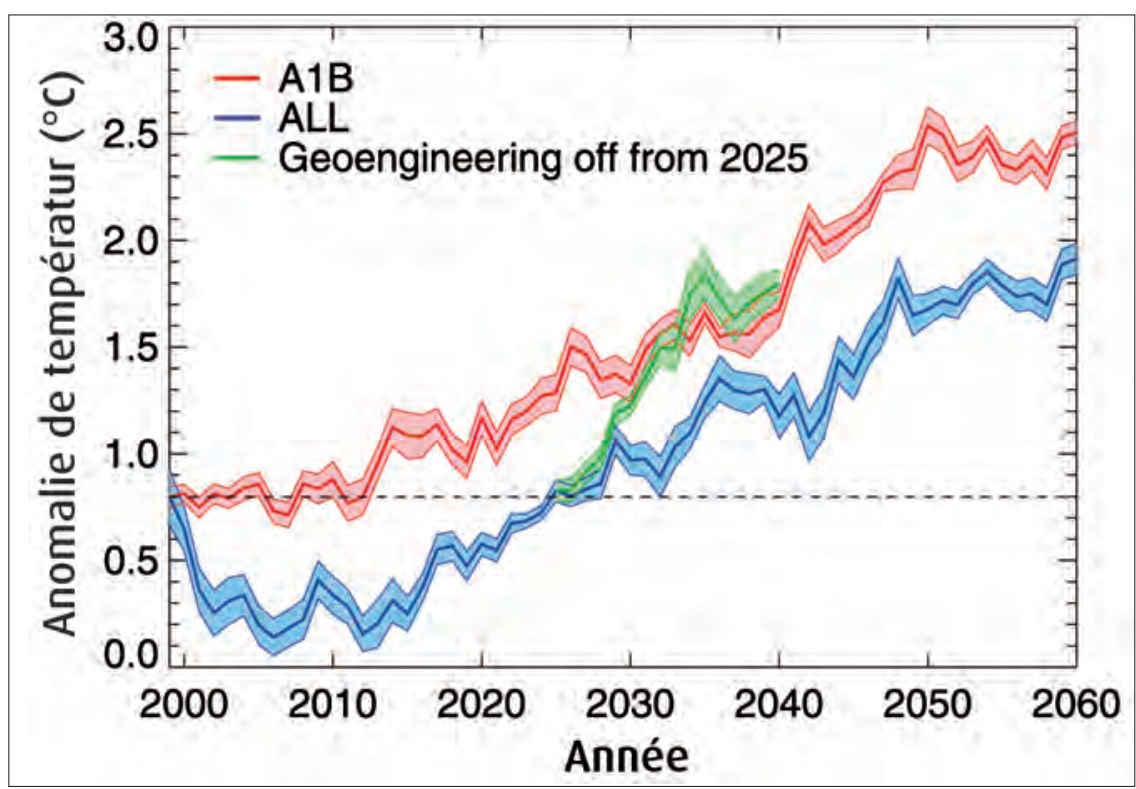

Figure 6 - Changement de température moyenne du globe en réponse au changement climatique pour un scénario A1B (courbe rouge). La courbe bleue est pour un scénario qui inclut une injection continue d'aérosols stratosphériques d'amplitude constante à partir de l'année 2000. La courbe verte montre l'impact d'une interruption brutale de cette injection en 2025. (D'après Jones et al. (2009). (C) UK Met Office, 2009) 
La gestion du rayonnement atmosphérique présente deux autres inconvénients de taille. D'une part, elle ne résout en rien le problème de l'acidification des océans due à l'augmentation de la concentration en $\mathrm{CO}_{2}$. D'autre part, elle doit se prolonger tant que les concentrations de $\mathrm{CO}_{2}$ ne sont pas redescendues à un niveau acceptable, ce qui prendrait des siècles, même au prix de réductions importantes des émissions futures (Boucher et al., 2009). Une interruption brutale de l'ingénierie climatique provoquerait un changement climatique rapide auquel il serait sans doute difficile de s'adapter (figure 6). On voit donc que la gestion du rayonnement atmosphérique pose un risque à long terme qu'il est difficile d'évaluer et de prendre en compte car les modèles socioéconomiques placent généralement d'autant moins de valeur dans le futur que celui-ci est lointain, une pratique connue sous le nom d'actualisation (Pearce et al., 2003).

\section{Ingénierie climatique et gestion des risques}

Le problème de l'ingénierie climatique peut être vu à travers le prisme de l'analyse et de la gestion des risques. Le changement climatique présente des risques auxquels les politiques d'adaptation ne pourront pas toujours répondre, comme, par exemple, l'augmentation attendue des événements météorologiques extrêmes, une diminution possible des rendements agricoles qui ne serait pas compensée par l'effet de fertilisation dû au $\mathrm{CO}_{2}$ atmosphérique, ou l'augmentation du niveau de la mer et le risque migratoire qu'il induit. Les politiques d'atténuation du changement climatique présentent aussi des risques, parmi lesquels on peut citer le coût économique d'une transition trop rapide vers les énergies renouvelables, un développement incontrôlé du nucléaire civil et le devenir du $\mathrm{CO}_{2}$ stocké dans les couches géologiques. L'ingénierie climatique, on l'a vu, n'est pas dépourvue de risques propres : conséquences inattendues et indésirables sur le climat, confiance démesurée dans la disponibilité future de technologies hypothétiques, changement climatique rapide lié à un arrêt brutal des techniques de gestion du rayonnement solaire, ou impacts climatiques d'une éruption volcanique majeure qui s'ajouterait à une injection continue d'aérosols stratosphériques... Mais il faut aussi reconnaître que l'ingénierie climatique pourrait permettre de limiter le risque d'un changement climatique important, particulièrement dommageable pour nos sociétés.

L'humanité peut agir sur le forçage radiatif en contrôlant les émissions de gaz à effet de serre ou en ayant recours à l'ingénierie climatique. L'humanité, en revanche, ne contrôle pas la sensibilité climatique mais on s'attend à ce que notre connaissance sur ce paramètre progresse, si bien que la trajectoire sur les émissions pourrait être ajustée au fil du temps. Une politique prudente, qui refuserait l'ingénierie climatique, nécessite d'atténuer rapidement les émissions jusqu'à ce qu'on en sache suffisamment pour décider à quel niveau de forçage radiatif il est acceptable de stabiliser le climat. Une telle politique nous prémunit contre tout risque de changement climatique, mais elle peut se révéler très coûteuse tant que la sensibilité climatique et les impacts climatiques futurs ne sont pas connus avec certitude. Une politique climatique imprudente peut, au contraire, mener à une situation où, malgré ses risques propres, la gestion du rayonnement atmosphérique serait l'unique solution pour réduire rapidement les impacts du changement climatique, s'ils devenaient insupportables. Le problème qui se pose à la société est donc un problème de gestion des risques dans un océan d'incertitudes.

\section{Quel cadre pour l'ingénierie climatique?}

On a vu que les techniques d'extraction atmosphérique des gaz à effet de serre ont probablement un coût élevé et, tout comme les politiques de réduction des émissions de gaz à effet de serre, leurs bénéfices ne s'inscrivent que dans la durée et à condition d'être menées à grande échelle. Rien ne sert à un pays de capturer du $\mathrm{CO}_{2}$ si les pays voisins continuent d'émettre à tout va. Il est donc clair que ces techniques n'ont de sens que dans le cadre d'une politique climatique internationale forte. Dans ce cas, l'extraction atmosphérique devient simplement un outil supplémentaire dans un ensemble de solutions pour réduire les émissions. En ce sens, la capture du $\mathrm{CO}_{2}$ atmosphérique fournit une borne supérieure au coût de réduction du $\mathrm{CO}_{2}$. Il est en effet inutile de capturer le $\mathrm{CO}_{2}$ atmosphérique tant qu'il reste des sources non mobiles émettant du $\mathrm{CO}_{2}$ concentré (telle une centrale thermique ou une unité d'industrie lourde). Mais on peut imaginer que l'extraction atmosphérique fournisse une alternative à certains secteurs très difficiles à décarboner (comme le trafic aérien, par exemple). Keith et al. (2006) ont par ailleurs montré que la possibilité de pouvoir extraire du $\mathrm{CO}_{2}$ atmosphérique dans le futur, à un certain coût, vient modifier les stratégies climatiques à long terme et donc les trajectoires de réduction des émissions. Mais, là encore, il ne faut pas oublier les incertitudes et les risques de ces techniques qui nécessiteront des ruptures technologiques encore hypothétiques pour devenir viables.

À l'inverse des techniques d'extraction du $\mathrm{CO}_{2}$, le coût opérationnel de la gestion du rayonnement solaire est relativement faible, si bien qu'on peut imaginer un scénario où un pays déciderait de déployer de l'ingénierie climatique de manière unilatérale. Mais les impacts, quant à eux, peuvent traverser les frontières. On se trouve là dans un flou juridique quasi total. Il existe bien un traité international, appelé « Convention sur l'interdiction d'utiliser des techniques de modification de l'environnement à des fins militaires ou toutes autres fins hostiles » (ou convention ENMOD), qui rassemble 74 pays signataires depuis 1978. Mais l'ingénierie climatique ne rentre pas directement dans le cadre de cette convention puisque son but n'est pas hostile. La convention autorise d'ailleurs explicitement les recherches et les expériences sur la modification du temps. La Convention sur la diversité biologique est un autre traité international qui a été adopté lors du Sommet de la Terre à Rio de Janeiro en 1992 et qui vise à préserver la biodiversité. Sa conférence des parties a approuvé un texte interdisant le déploiement de techniques d'ingénierie climatique en 2010 (CBD, 2010). Toutefois, ce texte n'est pas contraignant et autorise les expériences justifiées par les besoins de la recherche. La question se pose donc de savoir si un texte supplémentaire serait nécessaire pour encadrer ou interdire les activités de recherche sur l'ingénierie climatique. En tout état de cause, il sera important de distinguer les différentes techniques et de différencier la gouvernance souhaitée selon le type d'activité (simulation numérique, recherche en laboratoire, expérience de terrain de petite échelle, expérience de terrain de grande échelle, implémentation locale, régionale ou transnationale, etc.). On peut noter que les pays de 
l'Organisation maritime internationale (OMI) ont voté une interdiction de la fertilisation des océans, à l'exception d'expériences scientifiques de petite échelle (IMO, 2007), interdiction qui a ensuite été reprise par les parties à la Convention sur la diversité biologique (CBD, 2008).

\section{Conclusion}

Nous avons discuté ici des opportunités, des risques et des incertitudes associés à l'ingénierie climatique. Il est clair que l'ingénierie climatique ajoute une dimension supplémentaire au problème posé par le changement climatique et à la recherche d'une solution politique (Wigley, 2006). Les recherches effectuées jusqu'à maintenant indiquent qu'en l'état des choses, les techniques de capture du $\mathrm{CO}_{2}$ atmosphérique ont un potentiel limité ou alors comportent des risques importants. La gestion du rayonnement solaire, quant à elle, n'offre pas, pour le moment, une alternative sûre aux efforts de réduction des émissions de gaz à effet de serre. Elle ne résout en rien le problème de l'acidification des océans induit par l'augmentation de la concentration atmosphérique en $\mathrm{CO}_{2}$. Il est cependant difficile d'occulter l'ingénierie climatique compte tenu du risque climatique et de son potentiel à refroidir le climat. À titre personnel, je suis favorable à un programme de recherche sur l'ingénierie climatique qui permettrait d'évaluer les avantages, les désavantages et les risques de ces méthodes, de la manière la plus objective qui soit, dans le cas indésirable, mais pas impossible, où l'humanité en aurait besoin dans le futur. Un tel programme devra s'accompagner d'une réflexion éthique sur ces recherches, en particulier si des expériences de terrain sont envisagées. Seule une approche multidisciplinaire permettra d'aller au-delà des rapports nationaux déjà existants (Royal Society, 2009 ; GAO, 2011 ; IPCC, 2011) quant à l'évaluation de l'ensemble des aspects scientifiques et socio-économiques de l'ingénierie climatique.

\section{Remerciements}

Je voudrais remercier ici mes anciens collègues du Met Office, Andy Jones et Jim Haywood, avec qui j'ai commencé à travailler sur la problématique de l'ingénierie climatique. Les vues exposées ici ont été forgées par de nombreux échanges avec d'autres collègues et au travers de discussions dans le cadre du projet IMPLICC(1) et des travaux du Groupe intergouvernemental d'experts sur l'évolution du climat. Cette étude a bénéficié du soutien financier du projet FP7 EuTRACE(2).

(1) IMPLICC : Implications and risks of engineering solar radiation to limit climate change.

(2) FP7 EuTRACE : Framework Programme 7 European Trans-disciplinary Assessment of Climate Engineering.

\section{Bibliographie}

\footnotetext{
Akbari H., S. Menon et A. Rosenfeld, 2009 : Global cooling: increasing world-wide urban albedos to offset $\mathrm{CO}_{2}$. Climatic Change, 94, $275-286$.

Alterskjær K., J. E. Kristjansson et $\boldsymbol{\emptyset}$. Seland, 2012 : Sensitivity to deliberate sea salt seeding of marine clouds observations and model simulations. Atmospheric Chemistry and Physics, 12, 2795-2807

Angamuthu R., P. Byers, M. Lutz, A. L. Spek et E. Bouwman, 2010 : Electrocatalytic $\mathrm{CO}_{2}$ conversion to oxalate by a copper complex. Science, $327,313-315$

Baciocchi R., G. Storti et M. Mazzotti, 2006 : Process design and energy requirements for the capture of carbon dioxide from air. Chemical Engineering and Processing, 45, 1047-1058

Bala G., P. B. Duffy et K. E. Taylor, 2008 : Impact of geoengineering schemes on the global hydrological cycle. PNAS, 105, 7664-7669.

Ban-Weiss G. A. et K. Caldeira, 2010 : Geoengineering as an optimization problem. Environ. Res. Lett., 5, 034009.

Boucher 0., 1997 : L'influence climatique des aérosols. La Météorologie, 8e série, 17, 11-22.

Boucher 0., 2008 : Ozone stratosphérique, rayonnement ultraviolet et changement climatique. La Météorologie, 8e série, 63, 25-31.

- Boucher 0., J. Lowe et C. D. Jones, 2009 : Constraints of the carbon cycle on timescales of climate-engineering options. Climatic Change, 92, 261-273

- Boucher 0. et G. Folberth, 2010 : New Directions: Atmospheric methane removal as a way to mitigate climate change? Atmos. Environ., 44, 3343-3345.

- Caldeira K. et L. Wood, 2008 : Global and Arctic climate engineering: numerical model studies. Philos. Trans. Roy. Soc. A, 366, $4039-4056$.

- CBD, 2008 : Decisions adopted by the conferences of parties to the Convention on Biological Diversity at its ninth meeting. COP 9 Decision IX/16, Bonn, 19-30 May 2008.

CBD, 2010 : Decisions adopted by the conference of parties to the Convention on Biological Diversity at its tenth meeting. COP 10 Decision X/33.Biodiversity and climate - change, Nagoya, 18-29 October 2010.

- Crutzen P. J., 2006 : Albedo enhancement by stratospheric sulfur injections: A contribution to resolve a policy dilemma? Climatic Change, 77, 211-220.

- Davidson P., H. E. M. Hunt et C. J. Burgoyne, 2010 : Apparatus and method for transporting particles to the atmosphere. Patent Application GB 2476518 , filed 12.03.2010. Disponible sur http://www.ipo.gov.uk

- Davis S. J., K. Caldeira et H. D. Matthews, 2010 : Future $\mathrm{CO}_{2}$ emissions and climate change from existing energy infrastructure. Science, 329, 1330-1333.

- Evans J .R. G., E. P. J. Stride, M. J. Edirisinghe, D. J. Andrews et R. R. Simons, 2010 : Can oceanic foams limit global warming? Climate Res., $42,155-160$.

- Friedlingstein P., P. Cox, R. Betts, L. Bopp, W. von Bloh, V. Brovkin, P. Cadule, S. Doney, M. Eby, I. Fung, G. Bala, J. John, C. Jones, F. Joos, T. Kato - M. Kawamiya, W. Knorr, K. Lindsay, H. D. Matthews, T. Raddatz, P. Rayner, C. Reick, E. Roechner, K.-G. Schnitzler, R. Schur, K. Strassmann, A. J. Weaver, - C. Yoshikawa et N. Zeng, 2006 : Climate-carbon cycle feedback analysis: Results from the C4MIP model intercomparison. J. Climate, 19, 3337-3353.

- Friedlingstein P., R. A. Houghton, G. Marland, J. Hackler, T. A. Boden, T. J. Conway, J. G. Canadell, M. R. Raupach, P. Ciais et C. Le Quéré, 2010 : Update on - $\mathrm{CO}_{2}$ emissions. Nature Geoscience, 3, 811-812.

- GAO, 2011 : Technology assessment: Climate Engineering: Technical status, future directions and potential responses. U.S. Government Accountability Office, GA0-11-71, - 121 p., Washington, USA.

- Hamwey R. M., 2007 : Active amplification of the terrestrial albedo to mitigate climate change: An exploratory study. Mitigation and Adaptation Strategies for Global Change, 12, 419-439

- Heckendorn P., D. Weisenstein, S. Fueglistaler, B. P. Luo, E. Rozanov, M. Schraner, L. W. Thomason et T. Peter, 2009 : The impact of geoengineering aerosols - on stratospheric temperature and ozone. Environ. Res. Lett., 4, 045108.
} 
: Hegerl G. C., F. W. Zwiers, P. Braconnot, N. P. Gillett, Y. Luo, J. A. Marengo Orsini, N. Nicholls, J. E. Penner et P. A. Stott, 2007 : Understanding and Attributing : Climate Change. In: Climate Change 2007: The Physical Science Basis. Contribution of Working Group I to the Fourth Assessment Report of the Intergovernmental Panel on - Climate Change. [S. Solomon, D. Oin, M. Manning, Z. Chen, M. Marquis, K.B. Averyt, M. Tignor and H. L. Miller (eds.)], Cambridge University Press, Cambridge.

- IMO, 2007 : Convention on the Prevention of Marine Pollution by Dumping on Wastes and Other Matter, 1972 and its 1996 Protocol. Decisions of the 29th Consultative Meeting of the Contracting Parties, 5-9 November 2007.

- IPCC, 2005 : Intergovernmental Panel on Climate Change: Special report on carbon dioxide capture and storage. [B. Metz, O. Davidson, H. de Coninck, M. Loos and L. Meyer (eds)], Cambridge University Press, Cambridge.

- IPCC, 2007 : Intergovernmental Panel on Climate Change, Climate Change 2007: The Physical Science Basis. Contribution of Working Group I to the Fourth Assessment : Report of the Intergovernmental Panel on Climate Change. [S. Solomon, D. Qin, M. Manning, Z. Chen, M. Marquis, K. B. Averyt, M. Tignor, and H. L. Miller (eds.)], - Cambridge University Press, Cambridge.

: IPCC, 2011 : Expert Meeting Report on Geoengineering. [0. Edenhofer, C. Field, R. Pichs-Madruga, Y. Sokona, T. Stocker, V. Barros, Q. Dahe, J. Minx, K. Mach, - G.-K. Plattner, S. Schlomer, G. Hansen, M. Mastrandrea (eds.)], IPCC Working Group III Technical Support Unit, Potsdam Institute for Climate Impact Research, Potsdam, : Germany.

: Irvine P. J., A. Ridgwell et D. J. Lunt, 2010 : Assessing the regional disparities in geoengineering impacts. Geophys. Res. Lett., 37, doi:10.1029/2010GL044447.

Irvine P. J., A. Ridgwell et D. J. Lunt, 2011 : Climatic effects of surface albedo geoengineering. J. Geophys. Res., 116, doi:10.1029/2011JD016281.

- Jones A., J. Haywood et O. Boucher, 2009 : Climate impacts of geoengineering marine stratocumulus clouds. J. Geophys. Res., D10106, doi:10.1029/2008JD011450.

- Jones A., J. Haywood et $\mathbf{0}$. Boucher, 2010 : Geoengineering by stratospheric $\mathrm{SO}_{2}$ injection: results from the Met Office HadGEM2 climate model and comparison with the Goddard Institute for Space Studies ModelE. Atmospheric Chemistry and Physics, 10, 5999-6006.

Keith D., 2000 : Geoengineering the climate: History and prospect. Annual Review of Energy and the Environment, 25, 245-284.

Keith D., M. Ha-Duong et J. K. Stolaroff, 2006 : Climate strategy with $\mathrm{CO}_{2}$ capture from the air. Climatic Change, 74, 17-45.

Keith D. W., 2010 : Photophoretic levitation of engineered aerosols for geoengineering. PNAS, 107, 16428-1643.

Kellogg W. W. et S. H. Schneider, 1974 : Climate stabilization: for better or for worse? Science, 186, 1163-1172.

Kheshgi H. S., 1995 : Sequestering atmospheric carbon dioxide by increasing ocean alkalinity. Energy, 20, 915-922.

Kiehl J. T., 2007 : Twentieth century climate model response and climate sensitivity. Geophys. Res. Lett., 34, L22710, doi:10.1029/2007GL031383.

Korhonen H., K. S. Carslaw et S. Romakkaniemi, 2010 : Enhancement of marine cloud albedo via controlled sea spray injections: a global model study of the influence - of emission rates, microphysics and transport. Atmospheric Chemistry and Physics, 10, 4133-4143.

Kravitz B., A. Robock, G. Stenchikov, K. Taylor, O. Boucher, H. Schmidt et M. Schulz, 2011 : The Geoengineeering Model Intercomparison Project (GeoMIP) : Atmospheric Science Letters, 12, 162-167.

: Latham J., 1990 : Control of global warming? Nature, 347, 339-340

: Lehmann J., J. Gaunt et M. Rondon, 2006 : Bio-char sequestration in terrestrial ecosystems - A review. Mitigation and Adaptation strategies for global change, 11, 395-419.

Lenton T. M. et N. E. Vaughan, 2009 : The radiative forcing potential of different climate geoengineering options. Atmospheric and Chemistry Physics, 9, $5539-5561$.

Marchetti C., 1977 : On geoengineering and the $\mathrm{CO}_{2}$ problem. Climatic Change, 1, 59-68.

: McKay D. J. C., 2009 : Sustainable energy - without the hot air. UIT Cambridge Ltd, 372 p. Disponible en ligne à l'adresse http://www.withoutthehotair.com.

- Meinshausen M., S. J. Smith, K. Calvin, J. S. Daniel, M. L. T. Kainuma, J-F. Lamarque, K. Matsumoto, S. A. Montzka, S. C. B. Raper, K. Riahi, A. Thomson,

G. J. M. Velders et D. P. P. van Vuuren, 2011 : The RCP greenhouse gas concentrations and their extensions from 1765 to 2300. Climatic Change, 109, 213-241.

- Mercado L. M., N. Bellouin, S. Sitch, O. Boucher, C. Huntingford et P. Cox, 2009 : Changing contribution of diffuse radiation to the global land carbon sink. Nature, 458, 1014-1017.

: Mitchell D. L. et W. Finnegan, 2009 : Modification of cirrus clouds to reduce global warming. Environ. Res. Lett., 4, 04510.

New N., D. Liverman, H. Schroder et K. Anderson, 2011 : Four degrees and beyond: the potential for a global temperature increase of four degrees and its implications. Phil. Trans. Roy. Soc. A, 369, 6-19.

Partanen A.-I., H. Kokkola, S. Romakkaniemi, V.-M. Kerminen, K. E. J. Lehtinen, T. Bergman, A. Arola et H. Korhonen, 2012 : Direct and indirect effects of sea spray geoengineering and the role of injected particle size. J. Geophys. Res., 117, D02203, doi:10.1029/2011JD016428.

Pearce D., B. Groom, C. Hepburn et P. Koundouri, 2003 : Valuing the future, Recent advances in social discounting. World Economics, 4, 121-141.

President's Science Advisory Committee, 1965 : Restoring the quality of our environment. Report of the Environmental Pollution Panel, Washington, DC.

Pugh L. H. et W. W. Umbreit, 2009 : Anaerobic $\mathrm{CO}_{2}$ fixation by autotrophic bacteria, Hydrogenomonas and Ferrobacillus. Archives of Biochemistry and Biophysics, 115, 122-128.

Rasch P. J., P. J. Crutzen et D. B. Coleman, 2008 : Exploring the geoengineering of climate using stratospheric sulfate aerosols: The role of particle size. Geophys. Res. Lett., 35, L02809, doi:10.1029/2007GL032179.

: Richter R. (de) et S. Caillol, 2011 : Fighting global warming: The potential of photocatalysis against $\mathrm{CO}_{2}, \mathrm{CH}_{4}, \mathrm{~N}_{2} \mathrm{O}, \mathrm{CFCs}$, tropospheric $\mathrm{O}_{3}$, $\mathrm{BC}$ and other major contribu- tors to climate change. Journal of Photochemistry and Photobiology C: Photochemistry Reviews, 12, 1-19.

Ricke K. L., M. G. Morgan et M. R. Allen, 2010 : Regional climate response to solar-radiation management. Nature Geoscience, 3, 537-541.

- Ridgwell A., J. S. Singarayer, A. M. Hetherington et P. J. Valdes, 2009 : Tackling regional climate change by leaf albedo bio-geoengineering. Current Biology, $19,146-150$.

Robock A., L. Oman et G. Stenchikov, 2008 : Regional climate responses to geoengineering with tropical and Arctic $\mathrm{SO}_{2}$ injections. J. Geophys. Res., 113, D16101, doi:10.1029/2008JD010050.

: Robock A., A. Marquardt, B. Kravitz et G. Stenchikov, 2009 : Benefits, risks, and costs of stratospheric geoengineering. Geophys. Res. Lett., 36, L19703, doi:10.1029/2009GL039209.

Rogelj J., W. Hare, J. Lowe, D. P. van Vuuren, K. Riahi, B. Matthews, T. Hanaoka, K. Jiang et M. Meinshausen, 2011 : Emission pathways consistent with a $2^{\circ} \mathrm{C}$ global temperature limit. Nature Climate Change, 1, 413-418.

Royal Society, 2009 : Geoengineering the climate: science, governance and uncertainty. Report 10/09, $82 \mathrm{p}$.

: Salter S., G. Sortino et J. Latham, 2008 : Sea-going hardware for the cloud albedo method of reversing global warming. Philos. Trans. Roy. Soc. A, 366, $3989-4006$.

: Schmidt H., K. Alterskjær, D. Bou Karam, O. Boucher, A. Jones, J. E. Kristjansson, U. Niemeier, M. Schulz, A. Aaheim, F. Benduhn, M. Lawrence et

C. Timmreck, 2012 : Solar irradiance reduction to counteract radiative forcing from a quadrupling of $\mathrm{CO}_{2}$ : climate responses simulated by four earth system models. Earth Sys. Dynam., 3, 63-78. 
- Schuiling R. D. et P. L. de Boer, 2011 : Rolling stones; fast weathering of olivine in shallow seas for cost-effective $\mathrm{CO}_{2}$ capture and mitigation of global warming and ocean acidification. Earth Syst. Dynam. Discuss., 2, 551-568.

- Seitz R., 2011 : Bright water: hydrosols, water conservation and climate change. Climatic Change, 105, 365-381.

Shindell D., J. C. I. Kuylenstierna, E. Vignati, R. van Dingenen, M. Amann, Z. Klimont, S. C. Anenberg, N. Muller, G. Janssens-Maenhout, F. Raes, - J. Schwartz, G. Faluvegi, L. Pozzoli, K. Kupiainen, L. Höglund-Isaksson, L. Emberson, D. Streets, V. Ramanathan, K. Hicks, N. T. K. Oanh, G. Milly, M. Williams, V. Demkine et D. Fowler, 2012 : Simultaneously mitigating near-term climate change and improving human health and food security. Science, 335, 183-189.

- Thornton P. E., J.-F. Lamarque, N. A. Rosenbloom et N. M. Mahowald, 2007 : Influence of carbon-nitrogen cycle coupling on land model response to $\mathrm{CO}_{2}$ fertilization - and climate variability. Global Biogeochemical Cycles, 21, GB4018, doi:10.1029/2006GB002868.

- Tilmes S., R. Müller et R. Salawitch, 2008 : The sensitivity of polar ozone depletion to proposed geoengineering schemes. Science, 320, 1201-1204.

- Tilmes S., R. R. Garcia, E. D. Kinnison, A. Gettelman et P. J. Rasch, 2009 : Impact of geoengineered aerosols on troposphere and stratosphere. J. Geophys. Res., 114, D12305, doi:10.1029/2008JD011420

Urey H. C., 1952 : On the early chemical history of the Earth and the origin of life. Proc. Nat. Acad. Science USA, 38, 351-363.

- Wigley T. M. L., 2006 : A combined mitigation/geoengineering approach to climate stabilization. Science, 314, 452-454.

: Zeman F., 2007 : Energy and material balance of $\mathrm{CO}_{2}$ capture from air. Environ. Sci. Technol., 41, 7558-7563. 